\title{
Simulation of Abnormal Physiological Signals in a Phantom for Bioengineering Education
}

\author{
https://doi.org/10.3991/ijoe.v16i14.16941
}

\author{
Hélder Vieira, Nelson Costa, Joaquim Alves $\left.{ }^{(}{ }^{凶}\right)$, Luis Pinto Coelho \\ Instituto Superior de Engenharia do Porto, Porto, Portugal \\ jaa@isep.ipp.pt
}

\begin{abstract}
In clinical practice and in particular in the diagnostic process, the assessment of cardiac and respiratory functions is supported by electrocardiogram and auscultation. These exams are non-invasive, quick and inexpensive to perform and easy to interpret. For these reasons, this type of assessment is a constant in the daily life of a clinician and the information obtained is central to the decision-making process. Therefore, it is essential that during their training, students of health-related subjects acquire skills in the acquisition and evaluation of the referred physiological signals. Simulation, considering the technological possibilities of today, is an excellent preparation tool since it exposes trainees to near real contexts but without the associated risks. Hence, the simulation of physiological signals plays an important role in the education of healthcare professionals, bioengineering professionals and also in the development and calibration of medical devices. This paper describes a project to develop synchronized electrocardiogram (ECG), phonocardiogram (PCG) and breathing sounds simulators that aims to improve an existing phantom simulator. The developed system allows, in an integrated way, to generate normal and pathological signals, being contemplated several distinct pathologies. For engineering education, it is also possible to simulate the introduction of signal disturbances or hardware malfunctions.
\end{abstract}

Keywords-Biomedical signals, ECG, PCG, breathing sounds, simulation, modeling, Bioengineering education

\section{Introduction}

\subsection{Pathologies and Diagnostic methods}

Cardiovascular diseases (CVDs) are still the number one cause of death globally, taking approximately 17.9 million lives each year corresponding to an estimated $31.8 \%$ of all deaths worldwide, according to the World Health Organization (WHO) [1]. Respiratory diseases and lower respiratory infections, the third and fourth leading causes of death, represent 6.4 million deaths $(11.6 \%)$. All these, together, are responsible by $43.4 \%$ of deaths worldwide, with increased expression in developed countries. Improving these numbers requires reducing exposure to disease-inducing factors but also 
working in the fields of prevention and monitoring. Early diagnosis and appropriate therapy are often effective in eliminating or delaying the disease progression. Frequent monitoring of cardiac and respiratory functions can be conveniently performed by simple, non-invasive and cost-effective procedures using electrocardiogram (ECG), phonocardiogram (PCG) and auscultation. In fact, these simple examinations, provide crucial information for the diagnosis of the above-mentioned diseases and thus solid education and training in these topics are paramount.

The assessment of heart sounds, PCG, is a simple procedure that can be performed by healthcare practitioners as a routine. It can be useful to identify abnormal sounds for further investigation or to detect pathologies [2]. In addition, PCG analysis, often performed with intelligent and automated equipment, allows monitoring of the effect of certain cardiac drugs on the condition of the heart [3].

According to WHO data, more than $5 \%$ of the population suffers from respiratory problems, especially asthma. In addition to this evidence, over $14 \%$ of deaths in 2002 were caused by respiratory system-related diseases [4].

The use of auscultation to detect respiratory diseases is recurrent in clinical practice. This procedure is also non-invasive and allows rapid detection of pathologies or pathological patterns. Therefore, as the most basic semiotic method, the detection and identification of lung sounds becomes one of the first tests by which physicians guide themselves in defining the current diagnosis and the resulting therapy [4].

The ECG is an essential tool in the evaluation of the cardiac function. The detailed study and methodological analysis of the ECG components (waves, intervals and segments) form the basis of its interpretation [5], [6]. It has many applications in the clinical diagnosis and prognosis of CVDs, as well as in health assessment, biomedical recognition, fatigue studies, and other areas [7]-[9].

\subsection{Simulation in healthcare and engineering education}

Simulation is a valuable tool in the education and training of professionals, exposing students to near real situations while using controlled conditions. For medical students it can be an alternative to real patients providing replicated clinical scenarios where the trainee can practice and learn with a controlled sequence of tasks, while preventing dangerous situations. For biomedical engineering students it helps on gaining insight on physiological signals, understand their acquisition and associated problems, learn how to tackle common disturbances on the process. Overall, simulation allows the acquisition of skills through deliberate practice rather than an apprentice style of learning and students can be exposed to situations that occur on a daily basis but also to others that rarely happen in the real world [10], [11]. Additionally, this tool is also used in the testing and evaluation of the performance of acquisition systems and medical equipment, such as electrocardiographs [12].

The main purpose of this work it to design and develop two digital low-cost simulators that can be used as educational tools mainly for the medical field but also for biomedical engineering: one for the ECG and the other for both the PCG and the breathing sounds. Both simulators can generate normal and pathological signals allowing the replication of multiple clinical scenarios. 
Regarding ECG simulators, there are systems available designed to test medical equipment generally in the form of standalone hardware units that are capable of physically simulating ECG signals [13]. Besides such devices, there are software simulators designed to support the education of future health professionals without any physical output [14], [15]. The developed ECG simulator aims to combine the advantages of both systems. On one hand, a graphical user interface (GUI) allows the intuitive selection of signal parameters, control over the simulation and on-screen visualization of the simulated signal. On the other hand, the system is also capable of producing an electric signal that can be measured by acquisition equipment.

The Biomedical Simulation Center CHP-ICBAS in Portugal has currently a human simulator with some functionalities that brings it close to reality, such as movement of the chest, mouth, eyes and tongue, and $\mathrm{SpO}_{2}$ signal (oxygen saturation in the blood). However, it lacks some important aspects in addition to having no breathing or heart sounds, also has no ECG simulation and arterial blood pressure. Both simulators are meant to be incorporated in this existing phantom.

\section{Methods}

For both simulators, GUIs were developed with MATLAB®. The GUIs allow the user to adjust several signal parameters, control the simulation and to visualize the simulated signal graphically. Physical output can be produced allowing the user to measure/record the simulated signal. The block diagram of the general operation process of both simulators is represented in Fig 1.

Real signals (directly acquired from the human body), taken from publicly available databases, were used as templates for the signal generation processes of both simulators, hence providing a realistic output.

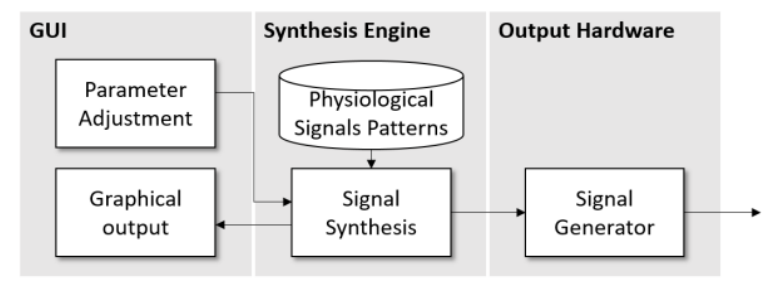

Fig. 1. Block diagram of the simulation process.

\subsection{ECG simulator}

Through the GUI, the ECG simulator allows the selection of several signal parameters, including:

- Beat type, having 6 possible selections, including normal and abnormal signals. 
- Heart rate (HR) in the interval from 30 to 200 beats per minute (bpm) with a resolution of $0.1 \mathrm{bpm}$. The resting cardiac activity can be classified regarding HR in bradycardia (when HR is below $60 \mathrm{bpm}$ ), tachycardia (HR higher than $100 \mathrm{bpm}$ ) and normal (HR value between $60 \mathrm{bpm}$ and $100 \mathrm{bpm}$ ) [16].

- Signal amplitude from 0 to $5 \mathrm{mV}$ with a resolution of $0.1 \mathrm{mV}$.

- Artefacts, having 2 possible selections (power line interference and baseline wander) and the respective intensity, from 0 to $1 \mathrm{mV}$ with a resolution of $0.1 \mathrm{mV}$.

A real ECG lead II normal signal with a sinus rhythm from a healthy person was obtained from the Physikalisch-Technische Bundesanstalt (PTB) diagnostic database available in the Physio Bank [17], [18]. The QRS complex, the P wave and the T wave were identified and separated.

The ECG Simulator generates lead II ECG signal with a heart rate (HR) dependent profile. Second order equations, determined in a previous work by Burke and Nasor [13], allow the calculation of the duration of each ECG component for a given HR value, generating an ECG signal profile that changes accordingly with HR, as can be seen in Fig 2.

The components extracted from the patterns database are then resampled (interpolated) according to the duration calculated using the mentioned equations. ECG segments (isoelectric sections between waves) are synthetized as arrays of zeros with the calculated length. The abnormal types are simulated through the change of parts of the sinus rhythm beat achieving a morphology similar to the real pathological characteristic signals, as can be seen in Fig 3 .
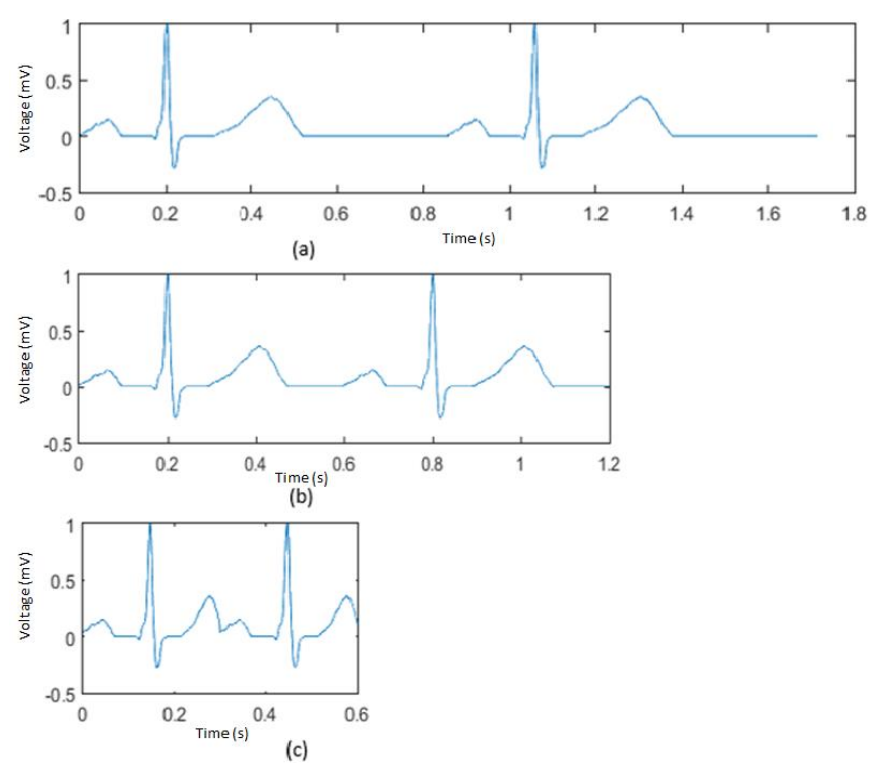

Fig. 2. ECG Tracings corresponding to 2 sinus rhythm complete cardiac cycles with different HR values where the timings were calculated using Burke and Nasor equations: (a) 70 bpm, (b) 100 bpm and (c) 200 bpm." 


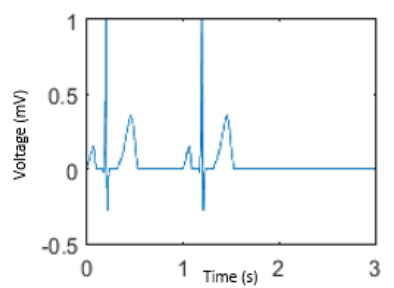

(a)

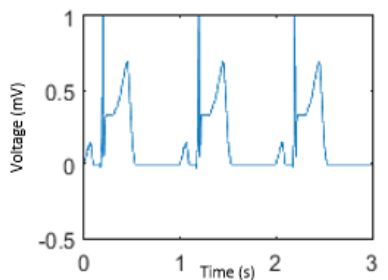

(c)

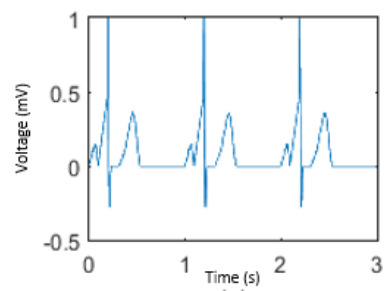

(e

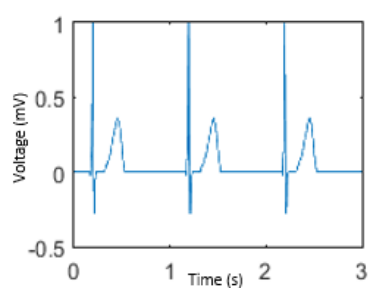

(b)

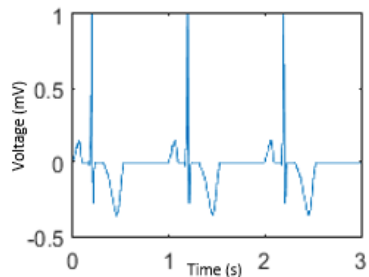

(d)

Fig. 3. Abnormal beat types: (a) 2nd degree type II sinoatrial block, (b) absent P waves (commonly seen in atrial fibrillation), (c) ST elevated Myorcardial Infarction, (d) Non-ST elevated Myocardial Infarction with T wave inversion and (e) Wolff-Parkinson-White Syndrome

The user can control the simulation through the GUI that can be seen in Fig 4. After the signal parameters are selected and the user clicks in the 'Simulate' button the simulation begins. The physical output is achieved using a low-cost open-source microcontroller board, Arduino UNO R3 and an electronic circuit, schematically presented in Fig 5. If the user selects the checkbox relative to physical output ('Output with Arduino') and indicates the serial port that the Arduino is connected to, the signal is transmitted to the Arduino board using serial communication via an USB port.

The Arduino UNO board can't directly generate an analog output, but it can output a pulse width modulation (PWM) signal that combined with a low-pass filter can be used to produce an analog signal with an amplitude range from 0 to $5 \mathrm{~V}$ [19].

Taking into account that the ECG signal generally has negative voltage components and the amplitude has to be adjusted to the value chosen by the user. The designed solution consists in:

1. Adjusting the ECG voltage values proportionally so that the baseline $(0 \mathrm{~V})$ from the synthetized signal corresponds to $2.5 \mathrm{~V}$ output from the low-pass filter. 
2. Amplifying, with a gain smaller than 1 (therefore attenuating), the difference between the signal from the filter and a DC signal of $2.5 \mathrm{~V}$, so that the maximum voltage corresponds to the value selected by the user.

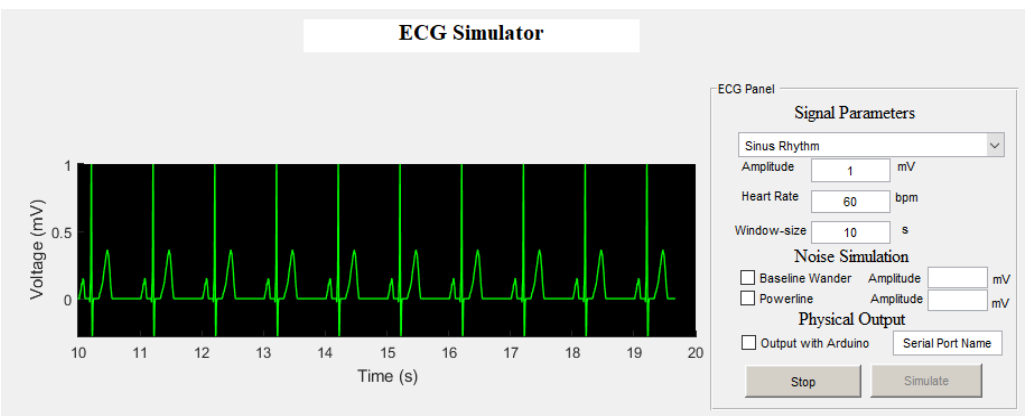

Fig. 4. GUI presenting a sinus rhythm ECG with $1 \mathrm{mV}$ of amplitude and a HR of $60 \mathrm{bpm}$.

The designed circuit, schematically represented in Fig 5, is essentially composed by two stages: (a) third-order active low pass filter (Sallen-Key topology) with a cutoff frequency of approximately $106 \mathrm{~Hz}$ (slightly higher than the typically clinical ECG maximum useful frequency of $100 \mathrm{~Hz}$ [20]) and (b) a differential amplifier that receives

the filtered signal and 2.5 V DC signal from a voltage divider (resistors R5 and R6) producing the resulting attenuated signal with a gain of 0.002 .

Signal artifacts are simulated using values from a sine function with the amplitude selected by the user and a frequency related to the chosen type: $50 \mathrm{~Hz}$ for the power line interference and $0.5 \mathrm{~Hz}$ for the baseline wander [21].

The low cost, low power consumption dual op-amp TL082 was used and the circuit was assembled in a breadboard.

The signal shown in the GUI is animated resembling an ECG signal being measured in real-time by a vital sign monitor with the traditional green tracing over a black background, providing an immersive educative experience to the student. A new simulation is possible clicking in the 'Stop' button in the GUI allowing the user to edit the signal parameters.

Fig 6 presents the entire simulator system including the computer running the GUI, the Arduino board, the electronic circuit in a breadboard and a digital oscilloscope (GW Instek GDS-2062) for acquiring the output signal. 


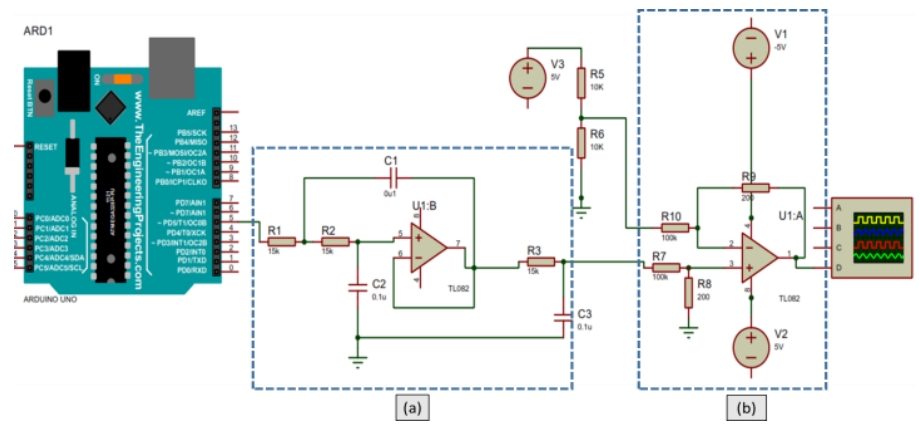

Fig. 5. Schematic representation of the ECG output hardware- Arduino board and a two-stage electronic circuit mainly composed by the (a) low pass filter and the (b) differential amplifier.

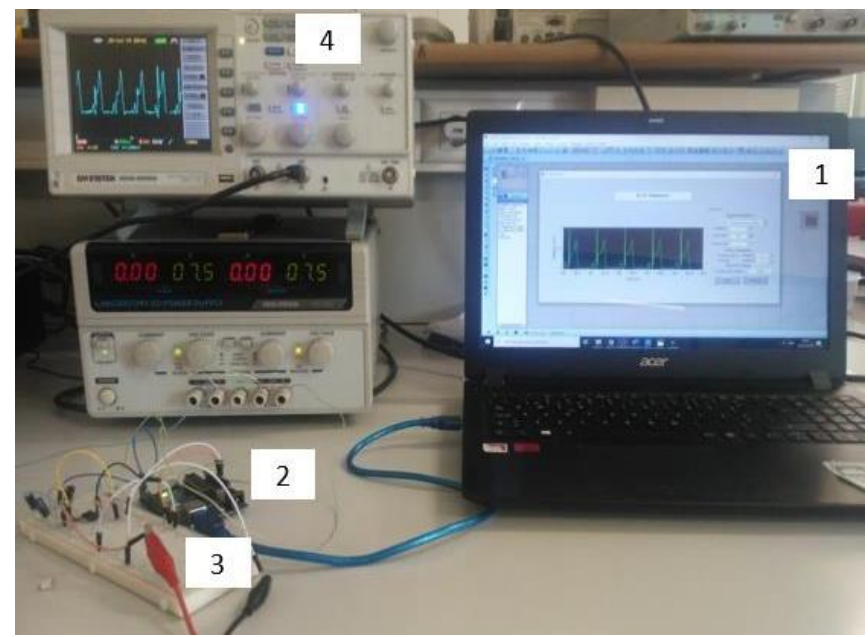

Fig. 6. The complete ECG simulation system: computer running the simulation software (1). The Arduino board producing a PWM signal (2) that is then converted by an electronic circuit (3) and a digital oscilloscope measuring the output signal (4).

\subsection{PCG and breathing sounds simulator}

Through the GUI, PCG and Breathing Sounds simulator allows the selection of several signal parameters, including:

- PCG signal type, which allows the user to choose between normal and pathological cardiac sounds such as premature atrial contraction sounds, 2nd degree type II sinoatrial block, mitral regurgitation and mitral stenosis.

- Heart rate (HR), allowing the user to adjust this value similarly to the ECG simulator.

- Amplitude, in which it is possible to adjust the sound intensity to the desired level. 
- Type of respiratory sound signal, in which the user can also simulate normal and pathological respiratory signals. The pathological signals implemented refer to apnea (absence of breathing activity), wheezing sounds and crackling sounds.

The Clini Surf database [22] allows the selection of the type of pathology desired and the place of auscultation. Regarding heart sounds, the apex of the heart was chosen as the place of auscultation for all extracted signals. The sampling frequency of the sounds available in this database is $44.1 \mathrm{kHz}$.

Initially, a cardiac sound recorded from a healthy subject was downloaded. Then the sound file was loaded and visualized graphically. Start and end positions of the first heart sound (S1) and the second heart sound (S2) were identified. The signals were isolated and stored in separated files.

Accordingly, Fig 7 shows the identification of the limits of the S1 (a) and S2 (b) heart sounds.

Breathing sounds were extracted from the Respiratory Sound Database created by two research teams in Portugal and Greece. This database was accessed through the Kaggle platform [23]. From this database, a normal record was extracted from which a segment corresponding to a complete respiratory cycle (inspiration and expiration) was identified and stored in a separate file. This sound's graphical representation is presented in Fig 8.

Two complete breathing cycles containing abnormal respiratory noises (crackles and wheezes, respectively) were identified from records in this database and stored separately.
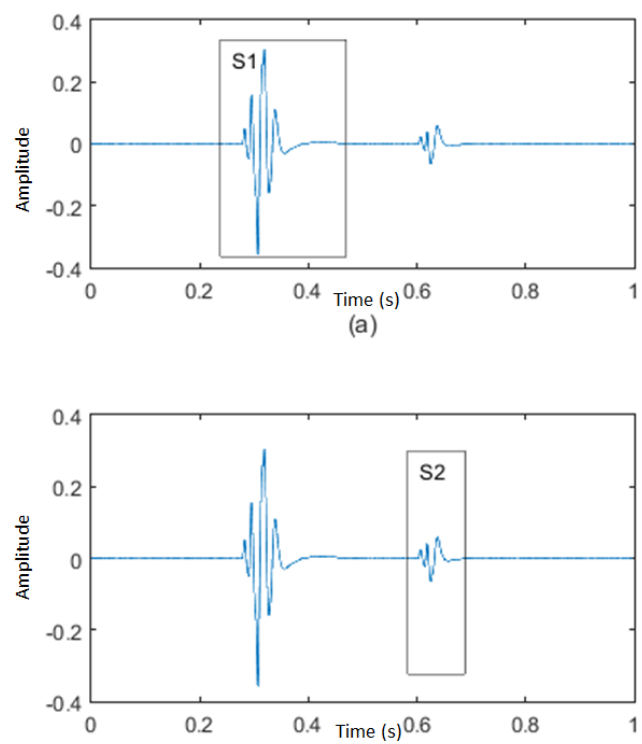

(b)

Fig. 7. Identification of the regions corresponding to the $S 1$ (a) and $S 2$ (b) heart sounds. 


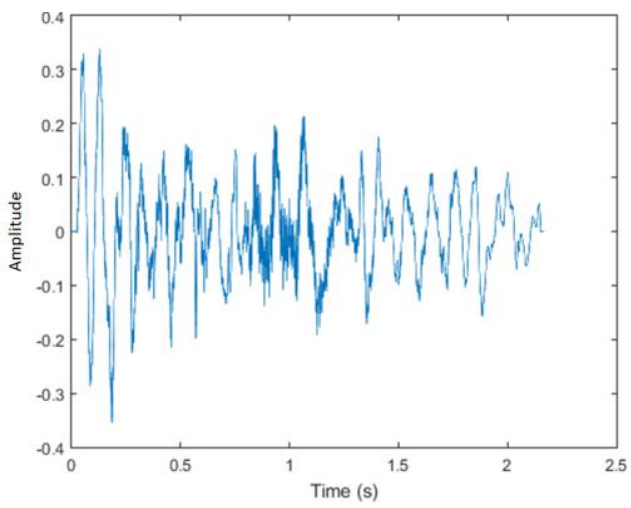

Fig. 8. Graph representing a complete cycle of normal respiratory sound.

Similarly, to the ECG simulation process, the PCG simulated signal is also influenced by the HR value. An HR dependent equation based on the work of Segaier et al. [24], is used allowing the calculation of the systolic period (Tsyst):

$$
T_{\text {syst }}=0.351-0.001 * H R
$$

In equation (1), Tsyst represents the systolic period in seconds and HR represents the heart rate value chosen by the user, in bpm.

The diastolic period is calculated through the difference between the total cardiac cycle duration $(60 / \mathrm{HR})$ and the Tsyst.

In the normal PCG type, the S1 and S2 are inserted in the beginning of the systolic and diastolic period, respectively. To the remaining samples of each cardiac phase is then attributed the value of zero (no sound) in order to achieve the calculated duration.

Abnormal PCG types available in the simulator can be divided in two major classes:

- Murmur based signal types, in which every cardiac cycle contains a murmur segment indicative of heart pathology.

- Probability based cardiac occurrences, in which a specific cardiac event can happen in some cardiac cycles and the occurrence decision is made randomly.

Murmur segments were extracted from the database, isolated and stored. Simulation of murmur based pathological signals consists of introducing the murmur in the proper position in the cardiac cycle. For example, in the case of the mitral stenosis type the isolated murmur can be seen in Fig 9. This murmur is placed in the diastolic phase right after the S2 sound, as shown in Fig 10.

Regarding the probability based PCG types (premature atrial contraction and the 2 nd degree type II sinoatrial block) in each cardiac cycle there is the possibility of event occurrence (in case of sinoatrial block there is no beat, in case of premature atrial contraction there is a premature beat making the diastole shorter).

This determination is made randomly using a function that generates an aleatory number in an interval ( 0 to 1$)$ and the generated number is then compared to a preset. 


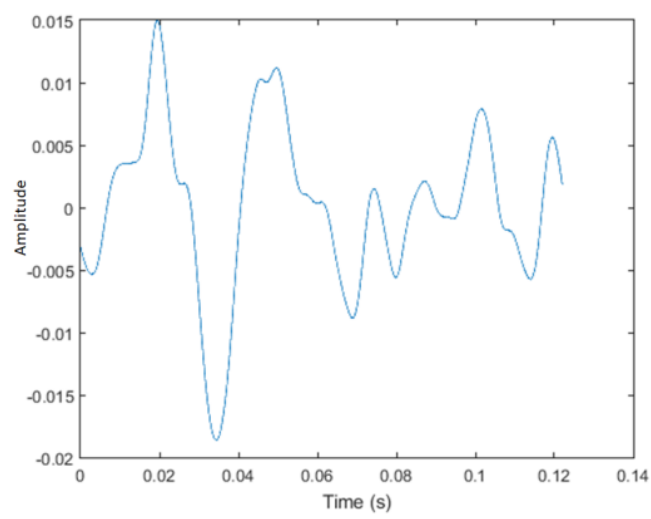

Fig. 9. Pathological signal portion characteristic of mitral stenosis.

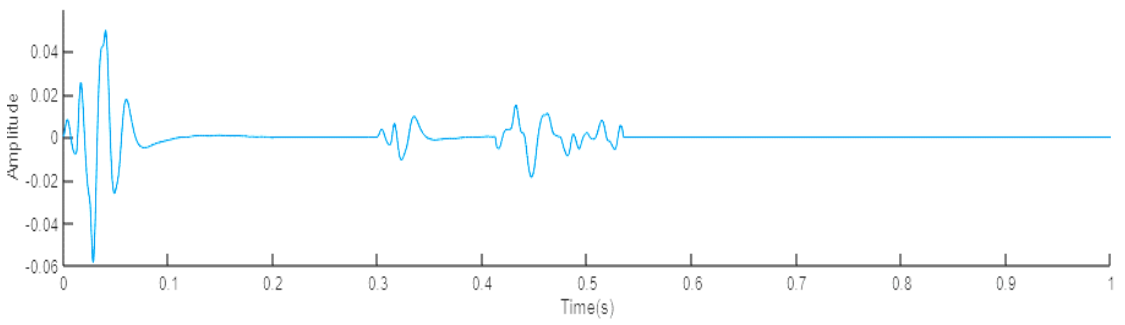

Fig. 10. Representative graphic of cardiac sound signal of mitral stenosis.

value $(0.1$, for example). If the generated number is smaller than the pre-established value, the event occurs.

In the graphical interface, one of the parameters allows the user to adjust the number of periods visible in each window.

Cardiac and respiratory signals are continuously displayed and outputted sonorously. To revalidate a new simulation, the user must change the input parameters and click the 'Simulate' button. When it reaches the end of the displayed graph, the current window is renewed, allowing a continuous simulation. Both sounds' amplitudes are adjustable and the simulated signals are physically generated simultaneously.

\section{$3 \quad$ Results and Discussion}

In order to evaluate the functionality of the developed simulators tests were performed for both the ECG and the PCG/Breathing sounds simulators.

\subsection{ECG simulator}

A digital oscilloscope (GW Instek GDS-2062) was used to record the electrical output produced by the Arduino and the electronic circuit. 
Fig 11 (a) shows the GUI output of a sinus rhythm ECG signal with a HR of $60 \mathrm{bpm}$ and a amplitude of $5 \mathrm{mV}$ and Fig 11(b) shows the signal output from the low-pass filter recorded in the oscilloscope. Both signals are very similar visually.

Several other tests were performed, changing all the signal parameters, producing generally satisfactory responses, corresponding to what was theoretically expected.

Figure 12 exhibits both the graphical (a) and physical (b) outputs from a normal sinus rhythm $60 \mathrm{bpm} 1 \mathrm{mV}$ amplitude ECG signal with a $0.4 \mathrm{mV}$ baseline wonder artifact. As can be seen, both outputs clearly show the presence of this artifact.

The differential amplifier output contained a substantial amount of noise most likely due to the fact that the circuit assembly was done in a breadboard the signal outputted from, the circuit implementation can be improved in the future by using a printed circuit board (PCB).

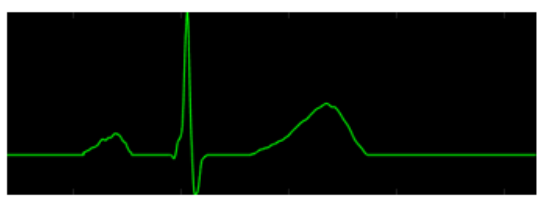

(a)

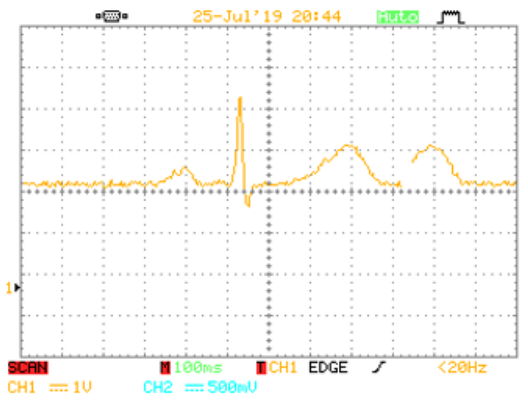

(b)

Fig. 11.Normal sinus rhythm ECG with $5 \mathrm{mV}$ of amplitude and $60 \mathrm{bpm}$ HR: (a) GUI representation and (b) physical output from the low-pass filter recorded with an oscilloscope 


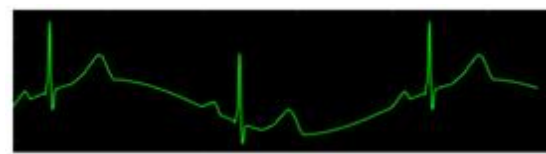

(a)

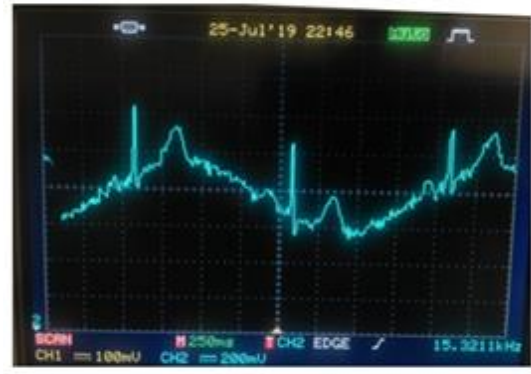

(b)

Fig. 12.Normal sinus rhythm ECG with $1 \mathrm{mV}$ amplitude and $60 \mathrm{bpm} \mathrm{HR}$ affected by $0.4 \mathrm{mV}$ amplitude baseline wonder artifact: (a) GUI output and (b) physical output from the low-pass filter

\subsection{PCG and breathing sounds simulator}

Fig 13 shows the results of a normal cardiac and respiratory sounds for an individual with a 60-bpm heart rate. The first and second heart sounds are well highlighted. Systole and diastole times are proportional, respecting the equation mentioned by Segaier et al. [18].

Regarding the graph of the respiratory sound signal, at the beginning of inspiration, high amplitude peaks stand out and, subsequently, a decrease in peak amplitude. It is further understood that the simulated cardiac and respiratory sounds are very identical to the sounds of individuals with no pathology. Thus, the results shown in Fig 13 are in accordance with what was theoretically expected.

Tests were performed for the other pathologies and the results obtained were generally satisfactory. 


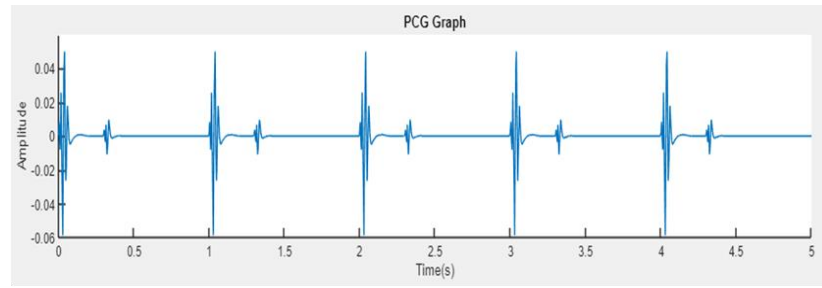

(a)

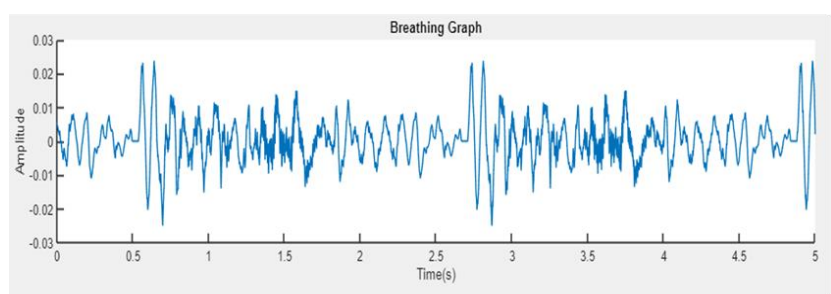

(b)

Fig. 13.Simulated sounds graphical representation cardiac. The displayed results have as fixed input parameters: cardiac signal amplitude $=0.10$; respiratory signal amplitude $=0.15$; number of cycles per window $=5$.

\section{Conclusion}

In this work, two simulators of physiological signals were developed with the purpose of enhancing the characteristics of an existing simulation dummy. All the development details have been thoroughly described, covering both hardware and software components. The main focus was to achieve maximum functionality with minimum costs. These simulators act as educational tools for health engineering and medical fields, allowing students of health-related subjects to acquire skills that are very difficult to acquire by other means. The advantages of using this type of simulators in education is well demonstrated by the number of commercial simulators available.

In future work, both simulators can be combined, synchronizing the respective signals while including interactions between pathologies or simulation contexts. Additionally, more pathologies can be added to increase the number of possible simulation scenarios and thus allowing to build more complex and diverse education/learning experiences.

\section{$5 \quad$ References}

[1] World Health Organization, Cardiovascular diseases (CVDs), May 17, 2017. https://www.who.int/news-room/fact-sheets/detail/cardiovascular-diseases-(cvds) (accessed Nov. 21, 2019). 
[2] A. Bourouhou, A. Jilbab, C. Nacir, and A. Hammouch, 'Heart Sounds Classification for a Medical Diagnostic Assistance', Int. J. Online Biomed. Eng. IJOE, vol. 15, no. 11, p. 88, Jul. 2019, https://doi.org/10.3991/ijoe.v15i11.10804

[3] M. F. Khan, M. Ateeq, and A. N. Qureshi, 'Computer Aided Detection of Normal and Abnormal Heart Sound using PCG', in Proceedings of the 2019 11th International Conference on Bioinformatics and Biomedical Technology, Stockholm Sweden, May 2019, pp. 94-99, https://doi.org/10.1145/3340074.3340086

[4] G. Jamieson, B. M. G. Cheetham, J. L. Moruzzi, and J. E. Earis, 'Digital signal processing of lung sounds', presented at the IEE Colloquium on DSP (Digital Signal Processing) in Instrumentation (Digest No.009), London, UK, 1992.

[5] J. Feldman and G. P. Goldwasser, 'Eletrocardiograma: recomendações para a sua interpretação', vol. 17, no. 4, p. 6, 2004.

[6] A. Kumar, J. Malik, and V. Kumar, 'Virtual Lab: Real-time Acquisition and Analysis of ECG Signal', Int. J. Online Eng. IJOE, vol. 7, no. 3, pp. 19-23, Jul. 2011, https://doi.org/10. 3991/ijoe.v7i3.1654

[7] S. A. Israel, J. M. Irvine, A. Cheng, M. D. Wiederhold, and B. K. Wiederhold, 'ECG to identify individuals', Pattern Recognit., vol. 38, no. 1, pp. 133-142, Jan. 2005, https://doi.org/10.1016/j.patcog.2004.05.014

[8] X.-L. Yang et al., 'The history, hotspots, and trends of electrocardiogram', p. 9.

[9] T. Rocha, S. Paredes, R. Cabiddu, R. Couceiro, P. Carvalho, and J. Henriques, 'A Tool For ECG Analysis as a Module of a Tele-Monitoring System', Int. J. Online Eng. IJOE, vol. 12, no. 04, p. 64, Apr. 2016, https://doi.org/10.3991/ijoe.v12i04.5260

[10] A. H. Al-Elq, 'Simulation-based medical teaching and learning', J. Fam. Community Med., vol. 17, no. 1, p. 35, 2010, https://doi.org/10.4103/1319-1683.68787

[11] H. Y. So, P. P. Chen, G. K. C. Wong, and T. T. N. Chan, 'Simulation in medical education', J. R. Coll. Physicians Edinb., vol. 49, no. 1, pp. 52-57, 2019: 10.4997/JRCPE.2019.112.

[12] Siemens, 'Simulation \& Testing for Medical Devices'. https://www.plm.automation.siemens.com/global/cz/industries/medical-devices-pharmaceuticals/simulation-test-medicaldevices.html. (accessed Nov. 27, 2019).

[13] M. J. Burke, and M. Nasor, 'An accurate programmable ECG simulator', J. Med. Eng. Technol., vol. 25, no. 3, pp. 97-102, Jan. 2001, 10.1080/03091900110051640.

[14] J.-C. Edelmann, D. Mair, D. Ziesel, M. Burtscher, and T. Ussmueller, 'An ECG simulator with a novel ECG profile for physiological signals', J. Med. Eng. Technol., vol. 42, no. 7, pp. 501-509, Oct. 2018, https://doi.org/10.1080/03091902.2019.1576788.

[15] E. Güney, Z. Ekşi, and M. Çakıroğlu, 'WebECG: A novel ECG simulator based on MATLAB Web Figure', Adv. Eng. Softw., vol. 45, no. 1, pp. 167-174, Mar. 2012, https://doi.org/10.1016/j.advengsoft.2011.09.005

[16] M. Escabí, 'Bio signal Processing', in Introduction to Biomedical Engineering, Elsevier, 2012, pp. 667-746. https://doi.org/10.1016/b978-0-12-374979-6.00011-3

[17] R. Bousseljot, D. Kreisler, and A. Schnabel, 'Nutzung der EKG-Signal daten bank CARDIODAT der PTB über das Internet', Biomed. Tech. Eng., pp. 317-318, Jul. 2009, https://doi.org/10.1515/bmte.1995.40.s1.317

[18] A. L. Goldberger et al., 'Physio Bank, Physio Toolkit, and Physio Net: Components of a New Research Resource for Complex Physiologic Signals', Circulation, vol. 101, no. 23, Jun. 2000, https://doi.org/10.1161/01.cir.101.23.e215 
[19] F. T. Omigbodun, 'Model Design of Low-Pass Filter of Integrated Pulse Width Modulation for Aircraft System Optimization', Int. J. Res. Appl. Sci. Eng. Technol., vol. 7, no. 6, pp. 1456-1465, Jun. 2019, https://doi.org/10.22214/ijraset.2019.6250

[20] K. A. Rosli, Mohd. H. Omar, A. F. Hasan, K. S. Musa, M. F. M. Fadzil, and S. H. Neu, 'Development of Electrocardiograph Monitoring System', MATEC Web Conf., vol. 150, p. 01013, 2018, https://doi.org/10.1051/matecconf/201815001013

[21] A. C. Vinzio Maggio, M. Paula, E. Laciar, and P. David, 'Quantification of Ventricular Repolarization Dispersion Using Digital Processing of the Surface ECG', in Advances in Electrocardiograms - Methods and Analysis, R. Millis, Ed. In Tech, 2012. https://doi.org/10. $\underline{5772 / 23050}$

[22] 'Clini Surf - Interactive learning modules for cardiology, endocrinology and ophthalmology’. http://clinisurf.elearning.aum.iml.unibe.ch/htmls/kardio.html?clinisurf|kardio (accessed 11 Aug. 29, 2019).

[23] 'Respiratory Sound Database | Kaggle'. https://www.kaggle.com/vbookshelf/respiratorysound-database. (accessed Jun. 04, 2020).

[24] M. El-Segaier, O. Lilja, S. Lukkarinen, L. Sörnmo, R. Sepponen, and E. Pesonen, 'Computer-Based Detection and Analysis of Heart Sound and Murmur', Ann. Biomed. Eng., vol. 33, no. 7, pp. 937-942, Jul. 2005, https://doi.org/10.1007/s10439-005-4053-3

\section{Authors}

Hélder Vieira received the Biomedical Engineering degree from the Polytechnic of Porto - Institute of Engineering (ISEP) in 2019. Currently working as an IT Consultant performing functions related to the Portuguese National Health System.

Nelson Costa has a Biomedical Engineering degree received from the ISEP (2019). Up until recently, worked as a medical equipment maintenance technician, performing clinical engineering and hospital management functions.

Joaquim Alves has a degree and master's in Electrical and Computer Engineering, at the Engineering Faculty of University of Porto (1994 and 1998) and PhD studies (2007) in Engineering Sciences, also at the University of Porto. Currently, he is Adjunct Professor at ISEP, teaching in this institution since 1997 in several degrees, in biomedical, instrumentation, metrology and physics subjects. Director of the Physics Department of ISEP since June 2016. Researcher in CIETI, in the areas of instrumentation and biomedical signal processing. Email: jaa@isep.ipp.pt

Luís Pinto Coelho has a degree and Msc in Electronics Engineering, at the Faculty of Engineering of Porto University, and a PhD in Telecommunications and Signal Processing from the University of Vigo, Spain (2012). He is currently a teacher at the Polytechnic Institute of Porto, collaborating in several degree plans and levels. His main research interests are natural interaction systems, signal and image processing for biomedical applications and machine learning.

Article submitted 2020-07-09. Resubmitted 2020-09-09. Final acceptance 2020-09-10. Final version published as submitted by the authors. 